\title{
Towards PPP-RTK: Ambiguity Resolution in Real-Time Precise Point Positioning
}

\author{
J., Geng, F.N., Teferle, X., Meng, A.H., Dodson \\ Institute of Engineering Surveying and Space Geodesy, the University of Nottingham, NG7 2TU, UK \\ Correspondence author: Jianghui Geng, isxjg@nottingham.ac.uk
}

\begin{abstract}
Integer ambiguity resolution at a single station can be achieved by introducing predetermined uncalibrated phase delays (UPDs) into the float ambiguity estimates of precise point positioning (PPP). This integer resolution technique has the potential of leading to a PPP-RTK (Real-Time Kinematic) model where PPP provides rapid convergence to a reliable centimeter-level positioning accuracy based on an RTK reference network. Nonetheless, implementing this model is technically subject to how rapidly we can fix wide-lane ambiguities, stabilize narrow-lane UPD estimates, and achieve the first ambiguity-fixed solution. To investigate these issues, we used seven days of $1-\mathrm{Hz}$ sampling GPS data at 91 stations across Europe. We find that at least 10 minutes of observations are required for most receiver types to reliably fix about $90 \%$ of wide-lane ambiguities corresponding to high elevations, and over 20 minutes to fix about $90 \%$ of those corresponding to low elevations. Moreover, several tens of minutes are usually required for a regional network before a narrow-lane UPD estimate stabilizes to an accuracy of far better than 0.1 cycles. Finally, for hourly data, ambiguity resolution can significantly improve the accuracy of epoch-wise position estimates from 13.7, 7.1 and $11.4 \mathrm{~cm}$ to $0.8,0.9$ and $2.5 \mathrm{~cm}$ for the East, North and Up components, respectively, but a few tens of minutes is required to achieve the first ambiguity-fixed solution. Therefore, from the timeliness aspect, our PPP-RTK model currently cannot satisfy the critical requirement of instantaneous precise positioning where ambiguity-fixed solutions have to be achieved within at most a few seconds. However, this model can still be potentially applied to some nearreal-time remote sensing applications, such as the GPS meteorology.
\end{abstract}

Keywords: Ambiguity resolution; Real time; Precise point positioning; Uncalibrated phase delay

\section{Introduction}

Precise point positioning (PPP) (Zumberge et al., 1997) can provide centimeter or even millimeter positioning accuracy with a single dual-frequency receiver of the Global Positioning System (GPS). Hence, PPP has been considered an effective post-event tool for the crustal-deformation monitoring (e.g., Melbourne et al., 2002), the ocean-tide measuring (e.g., King and Aoki, 2003), the atmospheric water vapor sensing (e.g., Bar-Sever et al., 1998; Rocken et al., 2005) and many other remote sensing applications (e.g., Jin and Komjathy, 2010). In real-time areas, PPP is being developed as a valuable tool in natural hazard early warning systems that can achieve homogeneous positioning accuracy on a global scale. One typical example is the Jet Propulsion Laboratory's GREAT (GPS Real Time Earthquake and Tsunami) Alert project which contains a prototype real-time PPP service for natural hazard prediction and monitoring 
(http://www.gdgps.net/products/great-alert.html), such as issuing tsunami alerts and assessing earthquake damages. However, this service currently cannot easily provide a centimeter-level positioning accuracy. One important reason for this deficiency is that integer resolution of carrier-phase ambiguities at a single station is normally ignored in PPP, thus inhibiting the further accuracy improvement of its position estimates.

Theoretically, these unresolved ambiguities are attributed to hardware-dependent non-integer uncalibrated phase delays (UPDs) originating in receivers and satellites (Blewitt, 1989; Ge et al., 2008). It is believed that these UPDs are very stable over time (e.g., Ge et al., 2008), and thus cannot be separated from integer ambiguities in a least squares adjustment, consequently destroying the integer property of ambiguities at a single station. Fortunately, a few authors have proposed that this integer property can be recovered if wide-lane and narrow-lane UPDs can be precisely predetermined using a network of reference stations. On the one hand, Ge et al. (2008) and Geng et al. (2009) computed wide-lane UPD estimates daily, but narrow-lane UPD estimates at a sub-daily frequency due to their temporal instability. By introducing these UPD corrections into float ambiguity estimates and performing integer resolutions, Ge et al. (2008) improved the daily positioning accuracy by about 10\%, and Geng et al. (2009) the hourly one by up to $70 \%$. On the other hand, Laurichesse and Mercier (2007) and Collins (2008) avoided estimating narrow-lane UPDs directly, but assimilated them into the satellite clocks. After applying such clocks, Collins et al. (2008) achieved an accuracy of better than $2 \mathrm{~cm}$ for $90 \%$ of hourly ambiguity-fixed position estimates.

Based on the advancements above, we can reasonably expect that real-time PPP can also be improved by applying ambiguity resolution. This application can potentially lead to an innovative PPP-RTK (Real-Time Kinematic) model where PPP provides rapid convergence of several seconds to a reliable centimeter-level positioning accuracy based on an RTK reference network (e.g., Bisnath and Gao, 2008; Wübbena et al., 2005), which can prevail against current realtime ambiguity-float PPP services (e.g., Dixon, 2006). In order to verify this concept, Laurichesse et al. (2008) and Mervart et al. (2008) reported that ambiguity resolution could improve the positioning accuracy in real-time PPP, but the convergence period to ambiguity-fixed solutions was usually over several tens of minutes at a mobile receiver. One reason for this long period is that smoothing noisy pseudorange observations for a reliable wide-lane ambiguity resolution usually requires a few tens of minutes; and the other is that the slowly changing geometry of visible satellites leads to an even longer period before a narrow-lane ambiguity resolution can be attempted (Laurichesse et al., 2008).

Hence, before implementing the PPP-RTK model above, we should carefully investigate at least three technical issues:

- Can we perform the wide-lane ambiguity resolution not only reliably, but also rapidly? This issue is critical to both the narrow-lane UPD determination at the server end and the narrow-lane ambiguity resolution at users; 
- Can narrow-lane UPD estimates stabilize rapidly to accurate values? To our knowledge, this issue has not been discussed in depth in recent publications, but it critically relates to how to rapidly provide usable narrow-lane UPD products for users;

- How does ambiguity resolution affect the positioning quality of real-time PPP? This issue has been preliminarily reported before, but more investigations over a relatively large spatio-temporal scale are necessary.

Note that other technical issues, such as the stochastic modeling (e.g., Jin et al., 2005) and the quality control (e.g., Han, 1997), are also important, but are hereafter ignored because they are beyond the scope of this paper.

In this paper, we therefore study the technical factors that affect the rapidity of both the wide-lane ambiguity resolution and the stabilization of narrow-lane UPD estimates. Then we investigate the performance of the narrow-lane ambiguity resolution in real-time PPP. In the following, Section 2 introduces the theories for UPD determination and ambiguity resolution in real time, and then presents a conceptual PPP-RTK model; Section 3 introduces the data and models used in this study; Section 4 shows the results and the pertinent discussion; finally, Section 5 summarizes the main points and the conclusions.

\section{Methods}

In this section, we briefly introduce the methods for the server solution to estimate UPDs, and for the user solution to perform ambiguity resolution. We then combine them to conceptually introduce a PPP-RTK model.

\subsection{Server solution}

For the server solution, we estimate UPDs by averaging merely the fractional parts of all pertinent ambiguity estimates at reference stations (Ge et al., 2008). Integer parts of these UPDs can be assimilated into integer ambiguities without degrading the positioning performance. Furthermore, we apply the between-satellite-difference (BSD) to undifferenced ambiguity estimates at each reference station to remove receiver-dependent UPDs, and thus a UPD estimate hereafter corresponds to a satellite pair. Additionally, we have to decompose ionosphere-free-observable ambiguities into widelane and narrow-lane ones. Note that applying this decomposition is because the ionosphere-free-observable ambiguities are not integers even though the UPDs have been corrected, and the wide-lane ambiguities can be fixed to integers using the Melbourne-Wübbena combination observations (Melbourne, 1985; Wübbena, 1985), but not because the wide-lane ambiguities can be relatively easily fixed (e.g., Teunissen, 1997).

In general, a wide-lane UPD $\phi_{w}^{i, j}$ between satellite $i$ and $j$ is estimated by 


$$
\phi_{w}^{i, j}=\left\langle\left[b_{w k}^{i, j}\right]-b_{w k}^{i, j}\right\rangle
$$

where $b_{w k}^{i, j}$ is a wide-lane BSD ambiguity estimate derived from the Melbourne-Wübbena combination observations at station $k ;[\cdot]$ denotes rounding to an integer; and $\langle\cdot\rangle$ denotes averaging all involved fractional parts. Ge et al. (2008) showed that wide-lane UPD estimates change within \pm 0.05 cycles within at least several days. Hence, wide-lane UPD products can be precisely predicted at an update rate of several days for real-time applications.

After $\phi_{w}^{i, j}$ is determined, the integer property of $b_{w k}^{i, j}$ can be recovered and then the narrow-lane UPD $\phi_{n}^{i, j}$ is determined by

$$
\phi_{n}^{i, j}=\left\langle\left[b_{n k}^{i, j}\right]-b_{n k}^{i, j}\right\rangle
$$

and

$$
b_{n k}^{i, j}=\frac{f_{1}+f_{2}}{f_{1}} b_{c k}^{i, j}-\frac{f_{2}}{f_{1}-f_{2}} n_{w k}^{i, j}
$$

where $f_{1}$ and $f_{2}$ denote the L1 and L2 frequencies, respectively; $b_{n k}^{i, j}$ is the narrow-lane BSD ambiguity estimate; $b_{c k}^{i, j}$ is the BSD ambiguity estimate for the ionosphere-free observable; $n_{w k}^{i, j}$ is the integer wide-lane BSD ambiguity. From Eq. (3), a bias in $b_{c k}^{i, j}$ will be amplified by about $70 \%$ in $b_{n k}^{i, j}$, very likely causing a pronounced variation of the fractional part of $b_{n k}^{i, j}$. In practice, Ge et al. (2008) reported that narrow-lane UPD estimates can change up to 0.4 cycles even within one day. They attributed this fluctuation to the inaccurate observation models which introduce some unknown biases, other than the UPDs, into ambiguity estimates, consequently leading to different fractional parts at reference stations and thus fluctuant narrow-lane UPD estimates. Hence, they chose to estimate narrow-lane UPDs every 15 minutes in order to obtain relatively high precisions. Likewise, we have to frequently estimate narrow-lane UPDs in our PPP-RTK model.

\subsection{User solution}

For the user solution, we use the UPD estimates above to correct the float wide-lane and narrow-lane BSD ambiguity estimates at a single station. Then we use sequential bias fixing (Dong and Bock, 1989) for the wide-lane ambiguity resolution, and LAMBDA (Least-squares AMBiguity De-correlation Adjustment) (Teunissen, 1994) method for the narrow-lane ambiguity resolution where the ratio test (Euler and Schaffrin, 1990) is used for ambiguity validation.

The integer property of a wide-lane BSD ambiguity $b_{w k}^{i, j}$ can be recovered by

$$
n_{w k}^{i, j}=b_{w k}^{i, j}+\phi_{w}^{i, j}
$$


Due to the noisy pseudorange observations used to estimate $b_{w k}^{i, j}, b_{w k}^{i, j}$ has to be smoothed by averaging over a specific time span. This span is mainly subject to the pseudorange measurement noise, multipath effects and atmospheric delays.

If $n_{w k}^{i, j}$ is successfully fixed to an integer, then the integer property of the corresponding narrow-lane BSD ambiguity $b_{n k}^{i, j}$ can be recovered by

$$
n_{n k}^{i, j}=\frac{f_{1}+f_{2}}{f_{1}} b_{c k}^{i, j}-\frac{f_{2}}{f_{1}-f_{2}} n_{w k}^{i, j}+\phi_{n}^{i, j}
$$

If $n_{n k}^{i, j}$ can also be successfully fixed to an integer, the ambiguity-fixed solutions can be obtained by highly weighting the following pseudo-observation in the estimation.

$$
b_{c k}^{i, j}=\frac{f_{1}}{f_{1}+f_{2}}\left(n_{n k}^{i, j}-\phi_{n}^{i, j}\right)+\frac{f_{1} f_{2}}{f_{1}^{2}-f_{2}^{2}} n_{w k}^{i, j}
$$

From Eq. (6), we note that narrow-lane UPD estimates, besides integer BSD ambiguity estimates, directly contribute to the ambiguity-fixed solutions. Hence, the accuracy of narrow-lane UPD estimates is crucial to the positioning accuracy.

\subsection{A conceptual PPP-RTK model}

Based on the methods above, Fig. 1 presents the data flowchart at both the server and user ends for a conceptual PPPRTK model. Compared with current real-time ambiguity-float PPP models, this model generates and disseminates not only the satellite orbits and clocks, but also the UPD products for users to achieve ambiguity resolution at a single station. Moreover, this model has to keep consistency among orbits, clocks and UPD products, rather than only between orbits and clocks (Geng et al., 2010).

Additionally, in Fig. 1, except the square denoting "orbit \& clock determination", the other four squares exhibit three modules which are crucial to an ambiguity resolution. We presume that wide-lane UPD products are easily known, and thus ignore their determination module in Fig. 1 for brevity. From the solid arrows in Fig. 1, the wide-lane ambiguity resolution is the prerequisite for both the narrow-lane UPD determination and the narrow-lane ambiguity resolution. We hereafter study these three modules which actually correspond to the bulleted questions in Section 1, by processing GPS data in a simulated real-time manner (i.e., forward data filtering without backward smoothing), but identifying cycle slips in advance and ignoring the communication delays which usually occur in practice.

\section{Data and models}

In this study, we used 24-hour 1-Hz GPS data at 91 stations from the European Reference Frame - Internet Protocol project (EUREF-IP) (Bruyninx, 2004) and the Ordnance Survey of Great Britain real-time network (Fig. 2). These data 
covered seven days from July 6th to 12 th in 2008. Particularly, EUREF-IP data reserve the gaps caused by communication or power interruptions, etc. (http://www.epncb.oma.be/). At these stations, we studied five receiver types including 9 Ashtech, 27 Javad, 21 Leica, 7 Topcon and 26 Trimble receivers. We hereafter simply use Type 1, 2, 3, 4 and 5 to represent them, but randomly change their sequence. Type-5 receivers contain six cross-correlation receivers and six receivers without choke-ring antennas.

For data modeling, the predicted parts of the IGS ultra-rapid satellite orbits and Earth rotation parameters were used from their release epochs until newer products were available. We applied the P1-C1 differential code biases, the absolute antenna phase centers, the phase wind-up corrections and the station displacement from the IERS conventions 2003 (McCarthy and Petit, 2004). We set a cut-off angle of $7^{\circ}$ for usable observations and applied an elevationdependent weighting strategy to observations at low elevations. Horizontal tropospheric gradients were estimated every 12 hours at the reference stations, whereas residual zenith tropospheric delays every 3 hours (Jin et al., 2007). We determined satellite clocks by fixing predicted orbits and station positions, whereas narrow-lane UPDs by fixing satellite orbits and clocks. About 30 stations were used to determine the satellite clocks, but all stations to determine UPDs, and finally six stations to simulate mobile users (Fig. 2). The PANDA (Positioning And Navigation Data Analyst) software (Shi et al., 2008) was used for this study.

\section{Results and discussion}

In this section, we analyze the rapidity of the wide-lane ambiguity resolution, the temporal stabilization of narrow-lane UPD estimates, and the performance of the narrow-lane ambiguity resolution, respectively.

\subsection{Rapidity of wide-lane ambiguity resolution}

The rapidity of the wide-lane ambiguity resolution is affected mainly by pseudorange measurement noise, multipath effects and atmospheric conditions. Empirically, the measurement noise is receiver-dependent, and the multipath effects and the atmospheric delays are very significant for satellites at low elevations. Hence, we divide ambiguities in terms of receiver types and an elevation angle of $15^{\circ}$ to illustrate the impacts of these factors on the wide-lane ambiguity resolution. We choose $15^{\circ}$ because cutoff angles are normally set below it to remove low-elevation observations which can degrade ambiguity estimates. Additionally, we need to clarify six points:

- For a satellite, we average its elevation angles over its observation periods to obtain time-mean values, each of which actually corresponds to an undifferenced ambiguity;

- For a being-observed satellite pair, its later-observed satellite, rather than the earlier-observed one, decides the starting time of forming a BSD ambiguity; 
- A round-off criterion of 0.25 cycles is applied to the bias fixing strategy by Dong and Bock (1989);

- If a wide-lane BSD ambiguity can be reliably fixed to an integer at a particular epoch, we actually mean that it can be fixed to the same integer at all subsequent epochs;

- The reliability of the wide-lane ambiguity resolution is quantified by the ratio of reliably-fixed ambiguities to all ambiguities;

- Integer values of fixed wide-lane BSD ambiguities in post-event data processing were used as the truth benchmarks.

\subsubsection{Ambiguities corresponding to high elevations}

In this study, ambiguities corresponding to high elevations are those derived from only the observations over an elevation angle of $15^{\circ}$. For each receiver type, Table 1 shows the mean time spent on the wide-lane ambiguity resolution and the distribution of all times. Each receiver type spends a mean time of between $100 \mathrm{~s}$ and $360 \mathrm{~s}$. On average, about $89.3 \%$ of all times are shorter than 600 s, and about $95.7 \%$ shorter than 1200 s. However, only $34.3 \%$ of all times are shorter than $20 \mathrm{~s}$ for all receiver types excluding Type 3 . These statistics suggest that $600 \mathrm{~s}$ of observations are required for most receiver types to reliably fix about $90 \%$ of wide-lane BSD ambiguities that correspond to elevations of over $15^{\circ}$

Interestingly, Type- 3 receivers show the shortest mean time of only $105 \mathrm{~s}$ that is almost over twice shorter than those of other receiver types; and even $86.4 \%$ of all times are shorter than $20 \mathrm{~s}$. This superiority might be related to the pseudorange smoothing strategies or the highly-qualified clocks applied within the Type-3 receivers. Hence, the rapid wide-lane ambiguity resolution within only several seconds can be potentially feasible for the Type-3 receivers.

Additionally, we investigate the impact of cross-correlation receivers and choke-ring antennas. Generally, crosscorrelation receivers suffer from relatively poor pseudorange quality and choke-ring antennas are useful in rejecting multipath-contaminated observations. In Table 1, the mean time is significantly reduced from 182 to $156 \mathrm{~s}$ by the exclusion of cross-correlation receivers (Type $5^{\mathrm{b}}$ ), and to $124 \mathrm{~s}$ by further exclusion of receivers without choke-ring antennas (Type $5^{\mathrm{c}}$ ). Moreover, due to these exclusions, the percentages of times that are shorter than 20,600 and $1200 \mathrm{~s}$ are steadily increased. Hence, these statistics demonstrate that cross-correlation receivers and the disuse of choke-ring antennas will prolong the time spent on the wide-lane ambiguity resolution.

\subsubsection{Ambiguities corresponding to low elevations}

Due to a small number of observations below an elevation angle of $15^{\circ}$, it is not appropriate to use only these observations to compute ambiguities corresponding to low elevations. Instead, they are represented by the wide-lane 
BSD ambiguities of which the later-observed satellites rise from below an elevation angle of $15^{\circ}$. For each receiver type, Table 2 shows the mean time spent on the wide-lane ambiguity resolution, the distribution of all times and the distribution of time-mean elevation angles for the later-observed satellites.

In Table 2, except Type-3 receivers, each receiver type spends a mean time of between 400 and 700 s. On average, only $73.0 \%$ of all times are shorter than $600 \mathrm{~s}$, and $87.6 \%$ shorter than $1200 \mathrm{~s}$. Compared with the statistics in Table 1 , these worse results can be attributed to the more severe multipath effects and the more turbulent atmospheric conditions at low elevations. Overall, we require over $1200 \mathrm{~s}$ of observations before fixing about $90 \%$ of wide-lane BSD ambiguities of which the later-observed satellites rise from below an elevation angle of $15^{\circ}$.

Alternatively, we propose to use the time-mean elevation angles of the later-observed satellites to decide when we can reliably fix a wide-lane BSD ambiguity. In Table 2, when wide-lane BSD ambiguities are fixed, over 90\% of time-mean elevation angles are smaller than $15^{\circ}$, and almost $100 \%$ smaller than $20^{\circ}$ for each receiver type. In practice, ambiguities corresponding to high elevations are preferred for integer resolutions, and the remnant ambiguities are usually left float to avoid risky fixing until their corresponding satellites arrive above a specific elevation angle, such as $15^{\circ}$. Hence, we believe that the time-mean elevation angle is more realistic as a threshold than the time span in fixing ambiguities corresponding to low elevations.

Additionally, from Table 2, the exclusion of cross-correlation receivers (Type $5^{\mathrm{b}}$ ) reduces the mean time by $22 \mathrm{~s}$, whereas the further exclusion of receivers without choke-ring antennas (Type $5^{\mathrm{c}}$ ) by $56 \mathrm{~s}$, suggesting the significant contribution of choke-ring antennas to mitigating multipath effects.

\subsubsection{High rapidity or high reliability}

Disappointingly, the results above demonstrate that the high rapidity of several seconds for the wide-lane ambiguity resolution cannot be normally achieved in our PPP-RTK model if high reliability of over $90 \%$ is required. In this case, we prefer the high reliability rather than the risky high-rapidity. We then carefully select the thresholds for both the time span and the time-mean elevation angle to decide when we can reliably fix a wide-lane BSD ambiguity. In Table 3 , for a threshold combination of $600 \mathrm{~s}$ and $15^{\circ}, 99.86 \%$ of all wide-lane BSD ambiguities can be reliably fixed. Both longer time and larger angle can improve this rate, but very slightly by not more than $0.05 \%$. Hence, we finally chose $600 \mathrm{~s}$ and $15^{\circ}$ as thresholds throughout this study.

\subsection{Temporal stabilization of narrow-lane UPD estimates}


Narrow-lane UPDs can be estimated once the wide-lane ambiguity resolution is achieved at the reference stations. Geng et al. (2009) reported that a narrow-lane UPD can be precisely determined for each full pass of a satellite pair over a regional network. In a real-time scenario, only up-to-present data, rather than all data during a full pass, can be used to estimate narrow-lane UPDs. As a result, narrow-lane UPD estimates may need some time to stabilize to accurate values. In this study, if the peak-to-peak amplitude during this stabilization decreases to smaller than 0.1 cycles, the narrowlane UPD estimates are considered sufficiently stable. Note that this threshold coincides with the accuracy requirement for narrow-lane UPD estimates by Ge et al. (2008).

\subsubsection{Fluctuant fractional parts}

Typically, Fig. 3 shows the fractional parts of a narrow-lane BSD ambiguity estimate and the two corresponding satellites' time-mean elevation angles every $100 \mathrm{~s}$. We can see that the fractional parts gradually stabilize to relatively stable values after a fluctuation during the initial phase. This initial phase lasts for a few tens of minutes, during which the peak-to-peak amplitude of the fractional parts is up to 0.2 cycles. Considering the elevation angles in Fig. 3, we argue that this fluctuation is related to the small elevation angles of the lower satellite during the initial phase.

In order to illustrate this argument, for all satellite pairs at all stations, Fig. 4 shows the fractional-part peak-to-peak amplitudes against the time-mean elevation angles of the lower satellites. Note that such an elevation angle is computed at only the epoch when the corresponding satellite pair has been observed for 10 or 30 minutes and the wide-lane ambiguity resolution has been achieved; moreover, the fractional-part estimates after only this epoch are used to compute the peak-to-peak amplitude and the narrow-lane UPD estimates. The blanks below $15^{\circ}$ in Fig. 4 are caused by the threshold of the time-mean elevation angle. In both Fig. 4(a) and 4(b), about 70\% of all peak-to-peak amplitudes are smaller than 0.1 cycles, and over $90 \%$ smaller than 0.25 cycles. Moreover, peak-to-peak amplitudes of larger than 0.5 cycles gather primarily below a time-mean elevation angle of $30^{\circ}$. Larger time-mean elevation angles lead to fewer peak-to-peak amplitudes that are larger than 0.1 cycles. Furthermore, prolonging the time span from 10 to 30 minutes significantly reduces the percentage of the peak-to-peak amplitudes of larger than 0.5 cycles from $0.8 \%$ to $0.4 \%$. Therefore, we demonstrate that large fractional-part fluctuations are closely related to small elevation angles, and usually occur during the initial observing phase of a satellite pair; additionally, the peak-to-peak amplitude is reduced when a satellite pair begins to be observed at a high elevation. These findings are likely related to carrier-phase multipath effects, imprecise satellite clock estimates, and residual tropospheric delays at low elevations.

\subsubsection{Fluctuant narrow-lane UPD estimates}


Large fractional-part fluctuations can degrade the performance of averaging fractional parts. For a being-observed satellite pair, different stations generate different fractional-part fluctuations, and thus we may have to average very different fractional parts to estimate a narrow-lane UPD. As a result, both the estimate's precision and accuracy can be degraded, compared with those when fractional parts have sufficiently stabilized. Hence, we need to use a robust averaging technique (e.g., $\mathrm{Xu}, 2005$ ), and involve as many stations as possible for this averaging. In this study, at least 10 stations are required.

For instance, Fig. 5 shows the fractional parts for a satellite pair, the number of involved stations, and the narrow-lane UPD estimates and their formal errors of $3 \sigma$ every $40 \mathrm{~s}$. During the initial phase, due to a satellite's rise from a low elevation with respect to our regional network, only a few stations observed this satellite pair. Consequently, large fractional-part fluctuations occur at many stations, leading to fluctuant narrow-lane UPD estimates of which the peakto-peak amplitude is over 0.1 cycles. During the intermediate phase when several tens of stations observe this satellite pair and most fractional parts have sufficiently stabilized, we obtain highly precise and very stable narrow-lane UPD estimates of which the peak-to-peak amplitude drops to smaller than 0.05 cycles. During the final phase, however, due to a satellite's gradual fall to a low elevation, the number of stations observing this satellite pair decreases, and thus the narrow-lane UPD estimates show slightly worse precisions and a slight fluctuation, but still within 0.05 cycles. Of particular note, large fluctuations of narrow-lane UPD estimates are mainly caused by satellites' rise from a low elevation with respect to our regional network. Hence, we believe that a global well-distributed network can reduce the fluctuation magnitudes.

Furthermore, if we begin to estimate narrow-lane UPDs when satellite pairs have been observed for at least 10 minutes, $69.0 \%$ of all estimates' peak-to-peak amplitudes are larger than 0.1 cycles. If the first 20 -minute or 50-minute estimates are removed, this percentage will drop to $38.2 \%$ or $15.1 \%$, respectively. Hence, for our regional network, over several tens of minutes are normally required before a narrow-lane UPD estimate stabilizes to an accuracy of far better than 0.1 cycles. Note that we require so high accuracy because narrow-lane UPDs are crucial to the ambiguity-fixed positioning accuracy (see Eq. (6)). However, for integer resolution only, this accuracy requirement can be relieved to 0.25 cycles, for example. Actually, $87.9 \%$ of all peak-to-peak amplitudes are smaller than 0.25 cycles if we begin to estimate narrow-lane UPDs when satellite pairs have been observed for 10 minutes. In this case, narrow-lane UPD products can be generated and disseminated once the wide-lane ambiguity resolution has been achieved. On the other hand, potential positioning biases caused by inaccurate narrow-lane UPD estimates can be mitigated in subsequent epochs by updating these estimates in the ambiguity-fixed solutions. In this study, we chose an update rate of $5 \mathrm{~s}$ due to the relatively rapid variation of narrow-lane UPD estimates during the initial phases. 


\subsection{Performance of narrow-lane ambiguity resolution}

Once we obtain the narrow-lane UPD estimates and achieve the wide-lane ambiguity resolution, we can attempt the narrow-lane ambiguity resolution to achieve ambiguity-fixed solutions in real-time PPP. Considering the time spent on the narrow-lane UPD determination, we ignore the first 30-minute data at all six test stations, and divide the remaining data into hourly files. Hence, we have about 161 hourly solutions at each test station during seven days. Daily position estimates are used as the ground truth to assess the accuracy of epoch-wise estimates.

Table 4 shows the overall statistics about the solution performance after the narrow-lane ambiguity resolution at each test station. All mean times that are spent on achieving ambiguity-fixed solutions are more than $900 \mathrm{~s}$, even up to $1200 \mathrm{~s}$, thus confirming the results reported by Laurichesse et al. (2008) and Mervart et al. (2008). Compared with the required 600-second period spent on the wide-lane ambiguity resolution, the narrow-lane ambiguity resolution significantly prolongs this period before we can achieve ambiguity-fixed solutions.

However, epoch-wise positioning accuracy is significantly improved after the narrow-lane ambiguity resolution. In Table 4, the RMS statistics against the ground truth for the East, North and Up components are reduced on average by $94.5 \%, 87.6 \%$ and $78.0 \%$ from $13.7,7.1$ and $11.4 \mathrm{~cm}$ to $0.8,0.9$ and $2.5 \mathrm{~cm}$, respectively. Moreover, Fig. 6 shows the $1-$ hour differences of epoch-wise position estimates from the ground truth for the East, North and Up components in both ambiguity-float and ambiguity-fixed solutions at station BSCN. We can see that ambiguity-float positions do not converge to centimeter-level accuracy within one hour, but the ambiguity-fixed ones achieve this after about $600 \mathrm{~s}$. Hence, according to the statistics above, we demonstrate that ambiguity resolution can speed up the convergence to centimeter-level accuracy of epoch-wise position estimates within a few tens of minutes.

\section{Summary and conclusions}

Ambiguity-fixed PPP can be achieved by introducing wide-lane and narrow-lane UPD products. Different from current real-time ambiguity-float PPP models, our PPP-RTK model additionally determines and disseminates UPD products to users who can then perform ambiguity resolution at a single station. In this study, we discussed three technical issues about this PPP-RTK model:

- Can we both rapidly and reliably fix wide-lane BSD ambiguities;

- Can we rapidly determine precise and accurate narrow-lane UPDs once we fix wide-lane BSD ambiguities;

- What are the benefits of fixing BSD ambiguities in real-time PPP?

First, we conclude that at least 10 minutes of observations are required for most receiver types to reliably fix about $90 \%$ of wide-lane BSD ambiguities corresponding to elevations of over $15^{\circ}$, and over 20 minutes to fix about $90 \%$ of those 
corresponding to elevations of below $15^{\circ}$. Moreover, receivers of cross-correlation types or without choke-ring antennas require longer periods than those of others. We propose two thresholds, namely 10 minutes for the time span and $15^{\circ}$ for the time-mean elevation angle, to decide when we can reliably fix a wide-lane BSD ambiguity.

Second, we conclude that several tens of minutes are usually required for a regional network before a narrow-lane UPD estimate stabilizes to an accuracy of far better than 0.1 cycles. Large fluctuations of narrow-lane UPD estimates are mainly caused by satellites' rise from a low elevation with respect to our regional network. However, we can still generate and disseminate narrow-lane UPD products once the wide-lane ambiguity resolution is achieved. These products are usable for integer ambiguity resolution, but we have to update them, every $5 \mathrm{~s}$ for example, in the ambiguity-fixed solutions in order to achieve highly accurate position estimates.

Finally, ambiguity-fixed solutions with centimeter-level positioning accuracy can be achieved within a few tens of minutes. For hourly data, ambiguity resolution significantly improves the positioning accuracy from 13.7, 7.1 and $11.4 \mathrm{~cm}$ to $0.8,0.9$ and $2.5 \mathrm{~cm}$ on average for the East, North and Up components, respectively.

For our PPP-RTK model, 10 minutes for the wide-lane resolution of high-elevation ambiguities, 10 minutes for the narrow-lane UPD generation and 5-second update rate are all acceptable at the server end. However, 10 minutes for the wide-lane ambiguity resolution and even longer period for the narrow-lane ambiguity resolution constitute the technical bottleneck which prohibits many real-time users who require instantaneous precise positioning from applying our PPPRTK model. However, this model is still useful in some remote sensing applications where the timeliness requirement on the first ambiguity-fixed solution is not as critical as that in the instantaneous positioning, such as the near-real-time GPS meteorology. For future technical development, rapid ambiguity resolution at a single station will be the key problem to be resolved before we can implement an instantaneous PPP-RTK model where ambiguity-fixed solutions can be achieved within a few seconds or even one second.

\section{Acknowledgements}

We would like to thank Wuhan University for the provision of PANDA software. Thanks also go to IGS, EUREF and Ordnance Survey of Great Britain for data provision. Two anonymous reviewers are acknowledged for their valuable comments which have improved this paper considerably.

\section{References}

Bar-Sever, Y.E., Kroger, P.M., Borjesson, J.A. Estimating horizontal gradients of tropospheric path delay with a single GPS receiver. J. Geophys. Res. 103(B3), 5019-5035, 1998.

Bisnath, S., Gao, Y. Current state of precise point positioning and future prospects and limitations, in: Sideris, M.G. (Ed.), Observing our Changing Earth. Springer-Verlag, New York, pp. 615-623, 2008. 
Blewitt, G. Carrier phase ambiguity resolution for the global positioning system applied to geodetic baselines up to 2000 km. J. Geophys. Res. 94(B8), 10187-10203, 1989.

Bruyninx, C. The EUREF permanent network: a multi-disciplinary network serving surveyors as well as scientists. GeoInformatics 7, 32-35, 2004.

Collins, P. Isolating and estimating undifferenced GPS integer ambiguities, in: Proceedings of ION National Technical Meeting, the Institute of Navigation Inc., Fairfax, US, pp. 720-732, 2008.

Collins, P., Lahaye, F., Héroux, P., Bisnath, S. Precise point positioning with ambiguity resolution using the decoupled clock model, in: Proceedings of ION GNSS 21st International Technical Meeting of the Satellite Division, the Institute of Navigation Inc., Fairfax, US, pp. 1315-1322, 2008.

Dixon, K. StarFire ${ }^{\mathrm{TM}}$ : A global SBAS for sub-decimeter precise point positioning, in: Proceedings of ION GNSS 19th International Technical Meeting of the Satellite Division, the Institute of Navigation Inc., Fairfax, US, pp. 22862296, 2006.

Dong, D., Bock, Y. Global positioning system network analysis with phase ambiguity resolution applied to crustal deformation studies in California. J. Geophys. Res. 94(B4), 3949-3966, 1989.

Euler, H.J., Schaffrin, B. On a measure of the discernibility between different ambiguity solutions in the statickinematic GPS mode, in: Schwarz, K.P., Lachapelle, G. (Eds.), Kinematic Systems in Geodesy, Surveying and Remote Sensing, IAG Symposium No. 107, Springer-Verlag, New York, pp. 285-295, 1990.

Ge, M., Gendt, G., Rothacher, M., Shi, C., Liu, J. Resolution of GPS carrier-phase ambiguities in precise point positioning (PPP) with daily observations. J. Geod. 82(7), 389-399, 2008.

Geng, J., Teferle, F.N., Shi, C., Meng, X., Dodson, A.H., Liu, J. Ambiguity resolution in precise point positioning with hourly data. GPS solut. 13(4), 263-270, 2009. doi: 10.1007/s10291-009-0119-2.

Geng, J., Teferle, F.N., Meng, X., Dodson, A.H. Kinematic precise point positioning at remote marine platforms. GPS solut. 2010. doi: 10.1007/s10291-009-0157-9.

Han, S. Quality-control issues relating to instantaneous ambiguity resolution for real-time GPS kinematic positioning. J. Geod. 71(6), 351-361, 1997.

Jin, S.G., Wang, J., Park, P.H. An improvement of GPS height estimates: stochastic modeling. Earth Planets Space 57(4), 253-259, 2005.

Jin, S.G., Park, J.U., Cho, J.H., Park, P.H. Seasonal variability of GPS-derived zenith tropospheric delay (1994-2006) and climate implications. J. Geophys. Res. 112, D09110, 2007. doi: 10.1029/2006JD007772.

Jin, S.G., Komjathy, A. GNSS reflectometry and remote sensing: new objectives and results. Adv. Space Res. 2010. doi: 10.1016/j.asr.2010.01.014.

King, M.A., Aoki, S. Tidal observations on floating ice using a single GPS receiver. Geophys. Res. Lett. 30(3), 1138, 2003. doi: 10.1029/2002GL016182.

Laurichesse, D., Mercier, F. Integer ambiguity resolution on undifferenced GPS phase measurements and its application to PPP, in: Proceedings of ION GNSS 20th International Technical Meeting of the Satellite Division, the Institute of Navigation Inc., Fairfax, US, pp. 839-848, 2007.

Laurichesse, D., Mercier, F., Berthias, J.P., Bijac, J. Real time zero-difference ambiguities fixing and absolute RTK, in: Proceedings of ION National Technical Meeting, the Institute of Navigation Inc., Fairfax, US, pp. 747-755, 2008.

McCarthy, D.D., Petit, G. IERS Conventions (2003). IERS technical note No. 32, Frankfurt am Main: Verlag des Bundes für Kartographie und Geodäsie, pp. 127, 2004.

Melbourne, W.G. The case for ranging in GPS-based geodetic systems, in: Goad, C. (Ed.), First International Symposium on Precise Positioning with the Global Positioning System, US Department of Commerce, pp. 373-386, 1985.

Melbourne, T.I., Webb, F.H., Stock, J.M., Reigber, C. Rapid postseismic transients in subduction zones from continuous GPS. J. Geohpys. Res. 107(B10), 2241, 2002. doi: 10.1029/2001JB000555.

Mervart, L., Lukes, Z., Rocken, C., Iwabuchi, T. Precise point positioning with ambiguity resolution in real-time, in: Proceedings of ION GNSS 21 st International Technical Meeting of the Satellite Division, the Institute of Navigation Inc., Fairfax, US, pp. 397-405, 2008.

Rocken, C., Johnson, J., Van Hove, T., Iwabuchi, T. Atmospheric water vapor and geoid measurements in the open ocean with GPS. Geophys. Res. Lett. 32(12), L12813, 2005. doi: 10.1029/2005GL022573.

Shi, C., Zhao, Q., Geng, J., Lou, Y., Ge, M., Liu, J. Recent development of PANDA software in GNSS data processing, in: Li, D., Gong, J., Wu, H. (Eds.) Proceedings of SPIE, the International Society for Optical Engineering, SPIE, Bellingham, WA., 7285, 72851S, 2008. doi: 10.1117/12.816261.

Teunissen, P.J.G. A new method for fast carrier phase ambiguity estimation, in: Proceedings of IEEE Position, Location and Navigation Symposium, IEEE Inc., New York, US, pp. 562-573, 1994.

Teunissen, P.J.G. On the GPS widelane and its decorrelating property. J. Geod. 71(9), 577-587, 1997.

Wübbena, G. Software developments for geodetic positioning with GPS using TI-4100 code and carrier measurements, In: Goad, C. (Ed.), First International Symposium on Precise Positioning with the Global Positioning System, US Department of Commerce, pp. 403-412, 1985.

Wübbena, G., Schmitz, M., Bagge, A. PPP-RTK: Precise point positioning using state-space representation in RTK networks, in: Proceedings of ION GNSS 18th International Technical Meeting of the Satellite Division, the Institute of Navigation Inc., Fairfax, US, pp. 2584-2594, 2005. 
$\mathrm{Xu}, \mathrm{P}$. Sign-constrained robust least squares, subjective breakdown point and the effect of weights of observations on robustness. J. Geod. 79(1-3), 146-159, 2005. doi: 10.1007/s00190-005-0454-1.

Zumberge, J.F., Heflin, M.B., Jefferson, D.C., Watkins, M.M., Webb, F.H. Precise point positioning for the efficient and robust analysis of GPS data from large networks. J. Geophys. Res. 102(B3), 5005-5017, 1997. 
Fig. 1. Data flowchart of a PPP-RTK model where cylinders denote data storage and squares denote data-processing modules; dashed arrows denote data input or output; and solid arrows denote processing sequence.

Fig. 2. Distribution of 1-Hz stations among which solid squares denote stations used for satellite clock determination; solid annotated triangles denote simulated mobile stations; and all symbols denote stations used for UPD determination.

Fig. 3. The fractional parts of a narrow-lane BSD ambiguity between PRN30 and PRN31 at station HOL2, and the satellites' time-mean elevation angles.

Fig. 4. The peak-to-peak amplitudes of narrow-lane fractional parts against the time-mean elevation angles for all satellite pairs after being observed for 10 (a) and 30 minutes (b).

Fig. 5. The narrow-lane fractional parts for a satellite pair PRN02 and PRN04, the number of involved stations, and the narrow-lane UPD estimates and their formal errors of $3 \sigma$. Note that the UPD estimates are offset by 0.4 cycles, and the fractional parts refer to the left axis.

Fig. 6. Differences of epoch-wise position estimates from the ground truth for the East, North and Up components in both the ambiguity-float and ambiguity-fixed solutions at station BSCN. Note that estimates outside $\pm 20 \mathrm{~cm}$ are not shown, and the three vertical lines denote the time when ambiguities are fixed. 